This is the final peer-reviewed accepted manuscript of:

Cagnone, S., Bartolucci, F. Adaptive Quadrature for Maximum Likelihood Estimation of a Class of Dynamic Latent Variable Models. Comput Econ 49, 599-622 (2017). DOI:10.1007/s10614-016-9573-4

The final published version is available online at: https://doi.org/10.1007/s10614016-9573-4

Rights / License:

The terms and conditions for the reuse of this version of the manuscript are specified in the publishing policy. For all terms of use and more information see the publisher's website.

This item was downloaded from IRIS Università di Bologna (https://cris.unibo.it/)

When citing, please refer to the published version. 


\title{
Adaptive quadrature for maximum likelihood estimation of a class of dynamic latent variable models
}

\author{
Silvia Cagnone and Francesco Bartolucci
}

Received: date / Accepted: date

\begin{abstract}
Maximum likelihood estimation of models based on continuous latent variables generally requires to solve integrals that are not analytically tractable. Numerical approximations represent a possible solution to this problem. We propose to use the Adaptive Gaussian-Hermite (AGH) numerical quadrature approximation for a particular class of continuous latent variable models for time-series and longitudinal data. These dynamic models are based on time-varying latent variables that follow an autoregressive process of order $1, \mathrm{AR}(1)$. Two examples are the stochastic volatility models for the analysis of financial time series and the limited dependent variable models for the analysis of panel data. A comparison between the performance of AGH methods and alternative approximation methods proposed in the literature is carried out by simulation. Empirical examples are also used to illustrate the proposed approach.
\end{abstract}

Keywords AR(1); Categorical longitudinal data; Gaussian-Hermite quadrature; Limited dependent variable models; Stochastic volatility model

\author{
S. Cagnone \\ Department of Statistical Sciences, University of Bologna (IT) \\ Tel.: +39-051-2098213 \\ E-mail: silvia.cagnone@unibo.it \\ F. Bartolucci \\ Department of Economics, University of Perugia (IT) \\ Tel.: +39-075-5855227 \\ E-mail: francesco.bartolucci@unipg.it
}




\section{Introduction}

Statistical models for the analysis of time-series and panel data often involve continuous time-varying latent variables. The dynamics of these latent variables is typically modeled by assuming that they follow an autoregressive process of order 1, denoted as usual by $\mathrm{AR}(1)$; see Frees (2004). In the analysis of financial time series data, this approach is adopted to study the volatility of returns that is time varying and autocorrelated. In particular, in the Stochastic Volatility (SV) model (Andersen, 1994; Taylor, 1994), the level of volatility is represented by a sequence of latent variables that follow a first order autoregressive process, as opposed to ARCH-type models Engle, 1982; Bollerslev, 1986).

A different example of data that may be effectively analyzed through latent autoregressive models is that of longitudinal/panel data where repeated observations on the same units are taken over time. Here the reason for using latent variables is to account for the non-observable heterogeneity between units. Within the variety of dynamic models discussed in the literature for panel data we consider, in particular, the Limited Dependent Variable (LDV) models for discrete data that are strictly related to generalized linear mixed models commonly used in the social sciences (Skrondal and Rabe-Hesketh, 2004; Hancock and Samuelson, 2008).

Both SV and LDV models have some attractive features. They can properly capture the variability in the data through an autoregressive latent structure and, at the same time, are more parsimonious than other models based on latent Markov chains (Bartolucci et al, 2013). Moreover, these models admit a general representation in the non-linear state space framework, but their estimation implies some computational difficulties as the time-varying latent variables must be integrated out from the likelihood function and an analytical solution for these integrals does not exist in general. In the literature, different solutions to this problem have been proposed, as we outline in the following.

For SV models, a simple estimation method is the generalized method of moments (Taylor, 1986). Moreover, Harvey et al (1994) proposed a quasi maximum likelihood approach based on the Kalman filter that has the advantage of not depending on specific distribution of the error terms. In the Bayesian context, Markov chain Monte Carlo techniques have been widely applied; see Jacquier et al (1994). Fridman and Harris (1998) proposed a direct maximum likelihood estimation of SV models using a non-linear Kalman filter algorithm. This algorithm is based on expressing the likelihood function as a nested sequence of one-dimensional integrals which are approximated by the Gauss Legendre numerical quadrature. Bartolucci and De Luca (2001, 2003) extended this approach by computing analytical first and second derivatives of the approximated likelihood. They applied a rectangular quadrature to approximate the integrals. A non-linear Kalman filter algorithm has been discussed, among others, by Tanizaki and Mariano (1998) and Durbin and Koopman (2002) in the general state space model framework. For the same SV model, Junii and Yoshihiko (2005) proposed a solution based on the Laplace approximation. All these studies show that the performance of the different approximation methods is highly sensitive to the values of the model parameters. 
For LDV models, a numerical integration solution was proposed by Heiss (2008) and consists in the use of a non-linear filter algorithm and the approximation of the resulting one-dimensional integrals through the Gauss Hermite $(\mathrm{GH})$ quadrature. $\mathrm{He}$ showed the higher performance of this method in comparison with other methods, among which the sparse grids integration (Heiss and Winschel, 2008), the non-linear particle filter (Fernández-Villaverde and Rubio-Ramírez, 2006), and the sequential importance sampling (Tanizaki and Mariano, 1998) in Bayesian settings. The superiority of this sequential $\mathrm{GH}$ quadrature over these alternative methods is due to the fastest convergence to the limiting values. However, it is known that GH based methods guarantee accurate parameter estimates when several quadrature points are used per each dimension. It is also important to consider that, for certain target functions, instability problems arise in the phase of maximization when the function is approximated through this quadrature method.

In this paper, we propose to use the Adaptive Gaussian Hermite (AGH) quadrature method to approximate the one-dimensional integrals involved in the non-linear filtering algorithm used for the estimation of SV and LDV models, when these models are specified within a general state space framework. AGH has been developed for the first time in the Bayesian context to compute posterior densities (Naylor and Smith, 1982; Liu and Pierce, 1994). More recently, it has been implemented and compared with other methods in a variety of random-effects and latent variable models; see, among others, Pinheiro and Bates (1995), Rabe-Hesketh et al (2002), Joe (2008), and Cagnone and Monari (2013). In all these studies this numerical method appeared to be superior to other quadrature methods, as it requires only few quadrature points to get accurate estimates, and it does not give rise to the risk of missing the maximum of the likelihood function, as it well captures the peak of the integrand involved in this function.

To our knowledge, AGH has not been previously applied for the estimation of dynamic latent variable models within the state space framework. In order to evaluate its performance for this class of models, we perform a wide simulation study under different conditions. For SV models, in particular, AGH is compared with alternative approximation methods discussed in the literature as well as with the Laplace approximation that can be viewed as a particular case of AGH when one quadrature point is used (Pinheiro and Bates, 1995). For LDV models, AGH quadrature is compared with the classical $\mathrm{GH}$ quadrature under different conditions.

The paper is organized as follows. In Section 2 we define the class of models of interest and, as particular cases, we describe SV and LDV models. In Section 3 model estimation is discussed, with particular attention to the AGH approximation. In Section 4 we illustrate the situations in which AGH outperforms the other quadrature methods in simple cases. Section 5 reports the results of the simulation study for both SV and LDV models. In Section 6 two applications on real data are illustrated. Some conclusions are provided in Section 7 


\section{State space specification of dynamic latent variable models}

In a time-series context, we let $y_{t}$ be the response variable observed at occasion $t$ with $t=1, \ldots, T$. For the case of panel data, in which we observe $n$ sample units at $T$ occasions, we extend this notation by denoting the response variable for unit $i$ at occasion $t$ by $y_{i t}, i=1, \ldots, n, t=1, \ldots, T$, and, since in this case covariates are also typically observed, we also denote by $\boldsymbol{x}_{i t}$ the vector of the covariates corresponding to $y_{i t}$.

In order to formulate dynamic latent variable models in the state space framework, we start from the more general case of panel data considering that time-series is a particular case with $n=1$. The proposed formulation is based on the following equations for $i=1, \ldots, n$ and $t=1, \ldots, T$ :

$$
\begin{aligned}
y_{i t} & =G\left(y_{i t}^{*}\right), \\
y_{i t}^{*} & =h\left(\alpha_{i t}, \boldsymbol{x}_{i t}, \varepsilon_{i t}\right), \\
\alpha_{i t} & =m\left(\alpha_{i, t-1}, \eta_{i t}\right),
\end{aligned}
$$

where $y_{i t}^{*}$ is a continuous unobservable variable underlying $y_{i t}$ introduced to deal with categorical response variable and $G(\cdot)$ is a parametric function, the specification of which depends on the nature of the observed variable. This function may involve thresholds in the presence of ordinal data. Moreover, $h(\cdot)$ and $m(\cdot)$ are known functions that can be linear or non-linear, whereas $\alpha_{i t}$ is a time dependent latent variable and $\varepsilon_{i t}$ and $\eta_{i t}$ are error terms assumed to be mutually independent. We refer to (1) as measurement equation and to (2) as transition equation. The former specifies the relationship between the manifest variables and the latent variables; the latter specifies the dependence between the latent variables over time. When both equations are linear, we obtain the classical linear state space models and the Kalman filter algorithm is usually used for model estimation. If one or both the equations are non-linear, we obtain a non-linear state space model.

In the following we illustrate two particular cases of non-linear state space models: SV models for the analysis of financial time series and LDV models for panel data.

\subsection{Stochastic volatility models for financial time series}

SV models are widely used for dealing with volatility in financial time series. These models represent the volatility by a sequence of latent variables following a first order autoregressive process and, therefore, they admit the following non-linear state space representation for $t=1, \ldots, T$ :

$$
\begin{aligned}
y_{t} & =\exp \left(\frac{1}{2} \alpha_{t}\right) \varepsilon_{t}, \quad \varepsilon_{t} \sim N(0,1), \\
\alpha_{t} & =\gamma+\alpha_{t-1} \rho+\eta_{t}, \quad \eta_{t} \sim N\left(0, \sigma_{\eta}^{2}\right) .
\end{aligned}
$$

In this case, $G(\cdot)$ is the identity function as the response variable is continuous. Moreover, $\exp \left(\alpha_{t}\right)$ is the volatility level underlying $y_{t}$; hence, the logarithm of the volatility level is assumed to follow an AR(1) process. 
2.2 Limited dependent variable models for panel data

Another class of models belonging to the general framework of non-linear state space models based on equations (1) and (2) is that of LDV models for the analysis of panel data. In this context, the latent variables are time and unit dependent random intercepts that allow us to account for the unobserved heterogeneity between subjects in a dynamic fashion. The non-linear state space representation of these models is given by

$$
\begin{aligned}
y_{i t} & =G\left(y_{i t}^{*}\right), \\
y_{i t}^{*} & =\boldsymbol{x}_{i t}^{\prime} \boldsymbol{\beta}+\alpha_{i t}+\varepsilon_{i t}, \\
\alpha_{i t} & =\alpha_{i, t-1} \rho+\eta_{i t}, \quad \eta_{i t} \sim N\left(0, \sigma_{\eta}^{2}\right),
\end{aligned}
$$

for $i=1, \ldots, n$ and $t=1, \ldots, T$ and where different distributions may be assumed for the error terms $\varepsilon_{i t}$.

In LDV models for panel data, the response variable $y_{i t}$ is typically discrete so that $G(\cdot)$ is not the identity function. In the binary case $G\left(y_{i t}^{*}\right)=I\left(y_{i t}^{*}>0\right)$, where $I$ is the indicator function, so that

$$
\lambda\left[p\left(y_{i t}=1 \mid \alpha_{i t}, \boldsymbol{x}_{i t}\right)\right]=\boldsymbol{x}_{i t}^{\prime} \boldsymbol{\beta}+\alpha_{i t},
$$

with $\lambda(\cdot)$ denoting the logit function or the inverse standard Normal cumulative function, resulting in a logit model or probit model, respectively.

If the response variables are ordinal with $J$ categories, we define a set of thresholds $\tau_{1} \leq \ldots \leq \tau_{J-1}$, such that

$$
G\left(y_{i t}^{*}\right)=j \Leftrightarrow \tau_{j-1}<y_{i t}^{*} \leq \tau_{j} \quad j=1, \ldots, J,
$$

with $\tau_{0}=-\infty$ and $\tau_{J}=+\infty$. Different models result according to the assumed distribution for the error terms $\varepsilon_{i t}$. For instance, assuming a logistic distribution leads to the well-known proportional odds model for cumulative probability functions (McCullagh, 1980):

$$
\log \frac{p\left(y_{i t} \leq j \mid \alpha_{i t}, \boldsymbol{x}_{i t}\right)^{\prime}}{p\left(y_{i t}>j \mid \alpha_{i t}, \boldsymbol{x}_{i t}\right)}=\tau_{j}-\alpha_{i t}-\boldsymbol{x}_{i t}^{\prime} \boldsymbol{\beta}, \quad j=1, \ldots, J-1 .
$$

\section{Model estimation}

Estimation of the models illustrated above may be carried out through the maximization of likelihood function

$$
L(\boldsymbol{\theta})=\prod_{i=1}^{n} f\left(y_{i 1}, \ldots, y_{i T}\right),
$$

where $\boldsymbol{\theta}$ is the vector of all model parameters which affects the manifest joint distribution of the observed variables $f\left(y_{i 1}, \ldots, y_{i T}\right)$. The latter can be expressed as

$$
f\left(y_{i 1}, \ldots, y_{i T}\right)=\int \cdots \int \prod_{t=1}^{T} f_{y}\left(y_{i t} \mid \alpha_{i t}\right) f_{\alpha}\left(\alpha_{i t} \mid \alpha_{i, t-1}\right) d \alpha_{i T} \cdots d \alpha_{i 1} .
$$


The above expression is based on the conditional independence assumption, according to which

$$
f\left(y_{i t} \mid y_{i 1}, \ldots, y_{i, t-1}, \alpha_{i 1}, \ldots, \ldots, \alpha_{i T}\right)=f\left(y_{i t} \mid \alpha_{i t}\right),
$$

and on the first order Markov assumption on the latent variables, according to which

$$
f_{\alpha}\left(\alpha_{i t} \mid y_{i 1}, \ldots, y_{i, t-1}, \alpha_{i 1}, \ldots, \ldots, \alpha_{i, t-1}\right)=f_{\alpha}\left(\alpha_{i t} \mid \alpha_{i, t-1}\right) .
$$

Notice that $f_{\alpha}\left(\alpha_{i 1} \mid \alpha_{i 0}\right) \equiv f_{\alpha}\left(\alpha_{i 1}\right)$. Moreover, the form of the densities $f_{y}\left(y_{i t} \mid \alpha_{i t}\right)$ and $f_{\alpha}\left(\alpha_{i t} \mid \alpha_{i, t-1}\right)$ depends on how the measurement equation (1) and the transition equation (2) are formulated.

Typically, computation and maximization of the likelihood function in (5) do not admit an analytical solution. An effective way to solve this problem is to apply nonlinear filter techniques that allow us to transform the multidimensional integral involved in expression (6) into a sequence of uni-dimensional integrals using the rules of conditioning as follows:

$$
f\left(y_{i 1}, \ldots, y_{i T}\right)=f\left(y_{i 1}\right) \prod_{t=2}^{T} f\left(y_{i t} \mid y_{i 1}, \ldots, y_{i, t-1}\right),
$$

where

$$
f\left(y_{i 1}\right)=\int f_{y}\left(y_{i 1} \mid \alpha_{i 1}\right) f_{\alpha}\left(\alpha_{i 1}\right) d \alpha_{i 1}
$$

and

$$
f\left(y_{i t} \mid y_{i 1}, \ldots, y_{i, t-1}\right)=\int f_{y}\left(y_{i t} \mid \alpha_{i t}\right) f\left(\alpha_{i t} \mid y_{1}, \ldots, y_{i, t-1}\right) d \alpha_{i t} .
$$

The density $f\left(\alpha_{i t} \mid y_{i 1}, \ldots, y_{i, t-1}\right)$ in expression $(8)$ can be obtained as follows:

$$
f\left(\alpha_{i t} \mid y_{i 1}, \ldots, y_{i t}\right)=\frac{f_{y}\left(y_{i t} \mid \alpha_{i t}\right) f\left(\alpha_{i t} \mid y_{i 1}, \ldots, y_{i, t-1}\right)}{f\left(y_{i t} \mid y_{i 1}, \ldots, y_{i, t-1}\right)},
$$

where

$$
f\left(\alpha_{i t} \mid y_{i 1}, \ldots, y_{i, t-1}\right)=\int f_{\alpha}\left(\alpha_{i t} \mid \alpha_{i, t-1}\right) f\left(\alpha_{i, t-1} \mid y_{i 1}, \ldots, y_{i, t-1}\right) d \alpha_{i, t-1} .
$$

The filtering algorithm consists in evaluating recursively formulas (8), (9), and (10).

Formulas (8) and (10) involve one-dimensional integrals that cannot be computed analytically and must be suitably approximated. A widely used method is the GaussHermite $(\mathrm{GH})$ quadrature method that is based on the following approximation:

$$
\int e^{-z^{2}} f(z) d z \simeq \sum_{k=1}^{q} w_{k} f\left(z_{k}\right)
$$

where $z_{k}$ are the zeros of the Hermite orthogonal polynomial $H_{k}, w_{k}$ are the correspondent weights, and $q$ is number of quadrature points (Davis and Rabinowitz, 1975). The approximation is exact if $f(z)$ is a polynomial of degree equal to $2 q-1$. 
In order to apply the GH to the integral (8) we rewrite it as

$$
f\left(y_{i t} \mid y_{i 1}, \ldots, y_{i, t-1}\right)=\int \frac{f_{y}\left(y_{i t} \mid \alpha_{i t}\right) f\left(\alpha_{i t} \mid y_{i 1}, \ldots, y_{i, t-1}\right) f_{\alpha}\left(\alpha_{i t}\right)}{f_{\alpha}\left(\alpha_{i t}\right)} d \alpha_{i t},
$$

where $f_{\alpha}(\cdot)$ is the density function of the marginal distribution of $\alpha_{i t}$ corresponding to a Normal density distribution with mean $\mu$ and variance $\sigma^{2}$. In particular, for SV models we have $\mu=\gamma /(1-\rho)$ and $\sigma^{2}=\sigma_{\eta}^{2} /\left(1-\rho^{2}\right)$; for LDV models we have $\mu=0$ and $\sigma^{2}=\sigma_{\eta}^{2} /\left(1-\rho^{2}\right)$. Denoting with $g\left(\alpha_{i t}\right)=f\left(\alpha_{i t} \mid y_{i 1}, \ldots, y_{i t-1}\right) / f_{\alpha}\left(\alpha_{i t}\right)$ and integrating over the standardized $\tilde{\alpha}=\frac{1}{\sigma}(\alpha-\mu)$, we obtain

$$
\begin{aligned}
f\left(y_{i t} \mid y_{i 1}, \ldots, y_{i, t-1}\right) & =\frac{1}{\sqrt{2 \pi}} \int f_{y}\left(y_{i t} \mid \sigma \tilde{\alpha}_{i t}+\mu\right) g\left(\sigma \tilde{\alpha}_{i t}+\mu\right) \exp \left(-\tilde{\alpha}_{i t}^{2} / 2\right) d \tilde{\alpha}_{i t} \\
& \simeq \sum_{k=1}^{q} f_{y}\left(y_{i t} \mid \sigma z_{k}^{*}+\mu\right) g\left(\sigma z_{k}^{*}+\mu\right) w_{k}^{*}
\end{aligned}
$$

where $z_{k}^{*}=\sqrt{2} z_{k}$ and $w_{k}^{*}=(1 / \sqrt{\pi}) w_{k}$. The same approximation can be applied to solve integral (10).

\subsection{Adaptive Gauss-Hermite quadrature}

An improved version of the GH approximation is given by the Adaptive Gauss Hermite (AGH) quadrature introduced in the Bayesian context with the aim of efficiently computing posterior densities if they are approximately Normal (Naylor and Smith, 1982). Essentially, AGH consists of adjusting the GH quadrature locations with the mean and the variance of the posterior density so that the nodes are more concentrated around the peak of the integrand, so that a better approximation of the function to be integrated is obtained.

For the particular class of models defined in Section 2, the AGH quadrature is obtained by multiplying and dividing the integral in (12) by the Normal density $\phi\left(\tilde{\alpha}_{i t}, \tilde{\mu}_{i t}, \tilde{\sigma}_{i t}\right)$. We have that

$$
\begin{aligned}
& f\left(y_{i t} \mid y_{i 1}, \ldots, y_{i, t-1}\right)= \\
& =\frac{1}{\sqrt{2 \pi}} \int \frac{f_{y}\left(y_{i t} \mid \sigma \tilde{\alpha}_{i t}+\mu\right) g\left(\sigma \tilde{\alpha}_{i t}+\mu\right) \exp \left(-\tilde{\alpha}_{i t}^{2} / 2\right)}{\phi\left(\tilde{\alpha}_{i t}, \tilde{\mu}_{i t}, \tilde{\sigma}_{i t}\right)} \phi\left(\tilde{\alpha}_{i t}, \tilde{\mu}_{i t}, \tilde{\sigma}_{i t}\right) d \tilde{\alpha}_{i t}
\end{aligned}
$$

such that, transforming the $\tilde{\alpha}_{i t}$ 's to standardized latent variables, the integral (14) is approximated as follows

$$
\begin{aligned}
& f\left(y_{i t} \mid y_{i 1}, \ldots, y_{i, t-1}\right) \simeq \\
& \simeq \tilde{\sigma}_{i t} \sum_{k=1}^{q} \frac{f_{y}\left(y_{i t} \mid \sigma \nu_{i t k}+\mu\right) g\left(\sigma \nu_{i t k}+\mu\right) \exp \left(-\nu_{i t k}^{2} / 2\right)}{\exp \left(-z_{k}^{* 2} / 2\right)} w_{k}^{*},
\end{aligned}
$$

where $\nu_{i t k}=\tilde{\sigma}_{i t} z_{k}^{*}+\tilde{\mu}_{i t}$.

Two different procedures can be used to determine $\tilde{\mu}_{i t}$ and $\tilde{\sigma}_{i t}$. The first one consists in approximating $\tilde{\mu}_{i t}$ with the mode of the integrand and $\tilde{\sigma}_{i t}$ with the standard 
deviation of the integrand at the mode (Liu and Pierce, 1994; Pinheiro and Bates, 1995; Schilling and Bock, 2005). The advantage of this approach is that the quadrature points are not involved in these computations. On the other hand, this method is very computationally demanding since it requires a numerical optimization. Moreover, when parameter estimates are obtained by using iterative algorithms, as in the cases dealt with in the present paper, the first two moments have to be computed at each step of the algorithm that, for this reason, is expected to be rather slow. An alternative method consists in computing the posterior mean and standard deviation (Naylor and Smith, 1982; Rabe-Hesketh et al, 2005). Although this method requires the use of quadrature points themselves, it is more robust to fat tailed distributions and it is faster when a sequential scheme is used. For these reasons we adopt the second procedure.

In more detail, following the second procedure, the computation of $\tilde{\mu}_{i t}$ and $\tilde{\sigma}_{i t}$ is iteratively obtained as follows:

1. Choose starting values $\tilde{\mu}_{i t}^{(0)}=0$ and $\tilde{\sigma}_{i t}^{(0)}=1$ so that $\nu_{i k}^{(0)}=z_{k}^{*}$.

2. Compute the log-likelihood for the $l$-th iteration

$$
\begin{aligned}
& \log L^{(l)}(\theta)=\log \prod_{i=1}^{n} f^{(l)}\left(y_{i 1}, \ldots, y_{i T}\right) \simeq \\
& \simeq \log \left[\prod_{i=1}^{n}\left(\prod_{t=1}^{T} \tilde{\sigma}_{i t}^{(l-1)} \sum_{k=1}^{q} \frac{f_{y}\left(y_{i t} \mid \sigma \nu_{i t k}^{(l-1)}+\mu\right) g\left(\sigma \nu_{i t k}^{(l-1)}+\mu\right) \exp \left(-\left(\nu_{i t k}^{(l-1)}\right)^{2} / 2\right)}{\exp \left(-z_{k}^{* 2} / 2\right)} w_{k}^{*}\right)\right], \\
& \text { with } g\left(\sigma \nu_{i t k}+\mu\right)=1 \text { for } t=1 .
\end{aligned}
$$

3. Update each node $\nu_{i t k}^{(l)}$ by computing the posterior mean and standard deviation as follows

$$
\begin{aligned}
& \tilde{\mu}_{i t}^{(l)}=\tilde{\sigma}_{i t}^{(l-1)} \sum_{k=1}^{q} \frac{\nu_{i t k}^{(l-1)} f_{y}\left(y_{i t} \mid \sigma \nu_{i t k}^{(l-1)}+\mu\right) g\left(\sigma \nu_{i t k}^{(l-1)}+\mu\right) \exp \left(-\left(\nu_{i t k}^{(l-1)}\right)^{2} / 2\right)}{f^{(l)}\left(y_{i t} \mid y_{i 1}, \ldots, y_{i t-1}\right) \exp \left(-z_{k}^{* 2} / 2\right)} w_{k}^{*}, \\
& \tilde{\sigma}_{i t}^{(l)}=\sqrt{\tilde{\sigma}_{i t}^{(l-1)} \sum_{k=1}^{q} \frac{\left(\nu_{i t k}^{(l-1)}\right)^{2} f_{y}\left(y_{i t} \mid \sigma \nu_{i t k}^{(l-1)}+\mu\right) g\left(\sigma \nu_{i t k}^{(l-1)}+\mu\right) \exp \left(-\left(\nu_{i t k}^{(l-1)}\right)^{2} / 2\right)}{f^{(l)}\left(y_{i t} \mid y_{i 1}, \ldots, y_{i t-1}\right) \exp \left(-z_{k}^{* 2} / 2\right)} w_{k}^{*}-\left(\tilde{\mu}_{i t}^{(l)}\right)^{2} .}
\end{aligned}
$$

Steps 2 and 3 are repeated until convergence is attained. Parameter estimation is obtained by quasi-Newton method. Thus the estimation algorithm consists in alternating one step of the quasi-Newton procedure to update parameter estimates and the iterative scheme proposed above to update the nodes of the adaptive numerical quadrature.

\section{Approximation of the probabilities with different numerical quadrature methods}

As described in Section 3, the GH quadrature should guarantee a sharp approximation of uni-dimensional integrals if an adequate number of quadrature points is chosen. This is because the true function is undistinguishable from its approximation based on a high order polynomial in most situations. However, if the integrand presents a 
narrow peak, GH does not provide sharp results even using a high number of quadrature points as it does not capture such a peak. On the other hand, in these cases the AGH quadrature guarantees a much sharper approximation of the true function.

For the class of models considered in this paper, the performance of the approximation methods strictly depends on the autocorrelation parameter $\rho$, in the case of the LDV model, and on the squared Coefficient of Variation of the volatility, $C V=\exp \left(\sigma_{\eta}^{2} /\left(1-\rho^{2}\right)\right)-1$, in the case of the SV model.

For illustrative purposes, consider a first simple case of a limited dependent variable model for panel data described in Section 2.2 for ordinal responses and $T=2$. Consider $J=4$ categories, thresholds equal to $\tau_{1}=-1.5, \tau_{2}=0, \tau_{3}=1.5$, and $\sigma^{2}=1$ without covariates. Suppose that for individual $i$ we observe category $j=3$. The probability reported in expressions (7) becomes

$$
\begin{aligned}
f\left(y_{i 1}\right) & =\int f_{y}\left(y_{i 1} \mid \alpha_{i 1}\right) f_{\alpha}\left(\alpha_{i 1}\right) d \alpha_{i 1}= \\
& =\int\left(p\left(y_{i 1} \leq 3 \mid \alpha_{i 1}\right)-p\left(y_{i 1} \leq 2 \mid \alpha_{i 1}\right)\right) \phi\left(\alpha_{i 1}\right) d \alpha_{i 1}
\end{aligned}
$$

where $p\left(y_{i 1} \leq 3\right)=\exp \left(1.5-\alpha_{i 1}\right) /\left(1+\exp \left(1.5-\alpha_{i 1}\right)\right)$, with $p\left(y_{i 1} \leq 2\right)$ defined accordingly, and $\phi(\cdot)$ stands for the standard Normal density. The behaviour of GH and AGH for the approximation of the integral in 16 is illustrated in Figure 1 (left picture) where the density $f_{y}\left(y_{i 1} \mid \alpha_{i 1}\right)$ is represented together with the relative $\mathrm{GH}$ and AGH nodes and weights for $q=21$. We observe that the integrand has a smooth shape and both methods approximate it very well and they perform similarly.

On the contrary, the integrand in expression 10 is not necessarily smooth due to the shape of the Normal density $f_{\alpha}\left(\alpha_{i 2} \mid \alpha_{i 1}\right)$ that strictly depends on the value of $\rho$. For the example here considered, equation (10) becomes

$$
f\left(\alpha_{i 2} \mid y_{i 1}\right)=\frac{1}{f\left(y_{i 1}\right)} \int \phi\left(\alpha_{i 2} ; \rho \alpha_{i 1},\left(1-\rho^{2}\right)\right) f_{y}\left(y_{i 1} \mid \alpha_{i 1}\right) \phi\left(\alpha_{i 1}\right) d \alpha_{i 1}
$$

where $\phi\left(\cdot ; \rho \alpha_{i 1},\left(1-\rho^{2}\right)\right)$ denotes the Normal density function with mean $\rho \alpha_{i 1}$ and variance $\left(1-\rho^{2}\right)$. In Figure 2 we show the product of the densities $\phi\left(\alpha_{i 2} ; \rho \alpha_{i 1},(1-\right.$ $\left.\left.\rho^{2}\right)\right) f_{y}\left(y_{i 1} \mid \alpha_{i 1}\right)$ in expression (17) for $\rho=0.5$ (left picture) and $\rho=0.95$ (right picture). While in the first case both the approximations perform well, in the second case only a small fraction of quadrature points are in the nonzero area and only AGH captures the peak of the integrand, outperforming GH. Clearly for $T$ greater than 2 , both probabilities (8) and (10) become more problematic to be approximated for high values of $\rho$.

[Fig. 1 about here.]

[Fig. 2 about here.]

[Fig. 3 about here.] 
As a second example, we consider a stochastic volatility model for financial time series described in Section 2.1 and, as in the previous example, we fix $T=2$ for simplicity. In this case, the probabilities in (8) and (10) become

$$
f\left(y_{1}\right)=\int f_{y}\left(y_{1} \mid \alpha_{1}\right) f_{\alpha}\left(\alpha_{1}\right) d \alpha_{1}=\int \phi\left(y_{1} ; 0, \exp \left(1 / 2 \alpha_{1}\right)\right) \phi\left(\alpha_{1}\right) d \alpha_{1}
$$

and

$$
f\left(\alpha_{2} \mid y_{1}\right)=\frac{1}{f\left(y_{1}\right)} \int \phi\left(\alpha_{2} ; \gamma+\rho \alpha_{1}, \sigma_{\eta}^{2}\right) f_{y}\left(y_{1} \mid \alpha_{1}\right) \phi\left(\alpha_{1}\right) d \alpha_{1}
$$

respectively. We consider two sets of parameters so that $C V=10$ and $C V=0.1$; the first one is $\gamma=-0.821, \rho=0.90$ and $\sigma_{\eta}=0.675$ and the second one is $\gamma=-0.706$, $\rho=0.90$, and $\sigma_{\eta}=0.135$.

In this case it is interesting to compare the performance of the AGH quadrature in approximating the probabilities in (18) and (19) with two quadrature based methods used in literature for SV models, the Gauss-Legendre quadrature (GLQ) approximation proposed by Fridman and Harris (1998) and a rectangular type quadrature (RQ) method proposed by Bartolucci and De Luca (2001).

The right picture of Figure 1 shows the Normal density $\phi\left(y_{1} ; 0, \exp \left(1 / 2 \alpha_{1}\right)\right)$ with relative AGH, GLQ, and RQ nodes and weights for $q=21$. As for the LVD models, also in this case all the quadrature approximations capture very well the area under the curve.

The approximation of the product $\phi\left(\alpha_{2} ; \gamma+\rho \alpha_{1}, \sigma_{\eta}^{2}\right) f_{y}\left(y_{1} \mid \alpha_{1}\right)$ in formula 19 depends on the value of $C V$. In the case of $C V=10$ (left picture, Figure 3 ) all the methods perform quite well, in particular AGH and RQ are more centered around the mode. On the other hand, for $C V=0.1$ (right picture, Figure 3), AGH and RQ perform similarly but only AGH captures the peak of the density whereas GLQ is clearly the worst method.

\section{Simulation study}

We carried out two simulation studies in order to evaluate the performance of AGH for LDV and SV models. In both cases the results are compared with other approximation methods discussed in the literature.

\subsection{Simulation study for the LDV models}

LDV model considered in this simulation study is the proportional odds model illustrated above. For this model, the behavior of AGH is compared with that of the classical GH approximation for increasing values of the correlation parameter $\rho=$ $(0.5,0.90,0.95)$, different combinations of time points $(T=5,10)$, and sample sizes $(n=500,1000)$. Five categories for the ordinal responses are chosen and the thresholds are fixed as $\tau_{1}=-1.65, \tau_{2}=-0.5, \tau_{3}=0.5$, and $\tau_{4}=1.65$. The remaining 
parameters $\beta$ and $\sigma$ are fixed to 1 . One covariate is generated from a stationary AR(1) process with autocorrelation parameter equal to 0.5 . The quadrature points chosen for AGH are $q=15,21$ and for GH are $q=21,51$. Under each simulation scenario, 500 samples were generated and the model parameters were estimated on the basis of the two approximation methods.

The comparison between the two different numerical approximations is assessed in terms of both accuracy of estimates and computational performance of the algorithms. For $T=5$ and $n=500$ the results are reported in Table 1 .

[Table 1 about here.]

For $\rho=0.5$, under both $\mathrm{GH}$ and AGH approximations the algorithm converges properly in almost all the samples $(\% \mathrm{cv})$. Moreover, the average number of function evaluations ( $n$ r feval) is quite similar for both methods. As for the rmse of the estimates, the best performances are obtained for $q=51$ with $\mathrm{GH}$, denoted with $\mathrm{GH}_{51}$ and for $q=21$ with $\mathrm{AGH}$, denoted with $\mathrm{AGH}_{21}$. They produce very close results for all the parameters estimates. In more detail, in terms of bias $\mathrm{AGH}_{21}$ is always better than $\mathrm{GH}_{51}$, in terms of rmse adaptive is better than the classical quadrature for $\beta$ and $\sigma$ and slightly worse for the autocorrelation parameter. Thus, with less than half quadrature points than those used with $\mathrm{GH}_{51}, \mathrm{AGH}_{21}$ produces very accurate estimates. Moreover, differently from GH, AGH is not affected by the choice of starting values. Nevertheless, the average computational time (av time in seconds) to convergence of the latter approximation is about three times that of the former, making GH preferable in this case. This is due to the iterative routine required for the computation of the posterior means and standard deviations at each iteration of the algorithm. However, it is possible to improve the speed of convergence of AGH by using a pseudo version of the AGH that consists in updating the quadrature nodes for each subject with the posterior means and standard deviations only at the first step of the algorithm, implying a consistently reduction of the computation time. This method was proposed by Rizopoulus (2012) for a class of linear mixed effect models for longitudinal and event time data. The author showed that, for the class of models considered, PseudoAGH produces accurate parameter estimates when proper starting values are used. Here we chose starting values by means of a multi-start strategy. The results of the PseudoAGH with $q=21$ are reported in the last column of Table 1 . We observe that the rmse of $\rho$ is the same as $\mathrm{AGH}_{21}$ whereas the rmse of the other parameter estimates are higher than the rmse obtained with $\mathrm{AGH}_{21}$. On the other hand, the computational time of PseudoAGH is noticeably lower than the standard AGH (the ratio is about 1 to 4 and in same cases about 1 to 5 ) and slightly lower than $\mathrm{GH}_{51}$.

In the cases of $\rho=0.90$ and $\rho=0.95$, the superiority of the adaptive procedure is undoubted. Indeed GH does not produce convergent solutions in all the generated samples due to instability problems. On the contrary, AGH performs very well even with $q=15$, being the rmse of all the estimates equal to those obtained for $q=21$. Moreover, in this case PseudoAGH presents the same rmse of all the parameter estimates as both $\mathrm{AGH}_{15}$ and $\mathrm{AGH}_{21}$ and, as before, reveals superior in terms of computational time. Thus, for high values of the autocorrelation parameters, PseudoAGH reveals the best method. The convergence problems under GH occurs in the cases of latent variables highly correlated over time determining very peaked integrands. 
The simulation results for $T=10$ and $n=500$ are reported in Table 2 As expected, in general the rmse of the estimates improve compared with those obtained in the previous scenario. As for GH, convergent solutions are obtained only for $\rho=$ 0.5 , whereas AGH performs well for all the values of the autocorrelation parameter even if the computational time is quite heavy. Also in this case, PseudoAGH seems to be the best compromise between GH and AGH, particularly in the case of high values of $\rho$ where, as before, the accuracy of the parameter estimates results similar and in same cases better than the standard adaptive rule. The reduction in the computational time results of the same magnitude as in the previous scenarios.

[Table 2 about here.]

The case of $T=5$ and $n=1000$ (Table 3) is the only one in which we obtain convergent solutions under $\mathrm{GH}$ also for high values of $\rho$.

[Table 3 about here.]

However, for $\rho=0.90$, even if $\mathrm{GH}_{51}$ performs similarly to $\mathrm{AGH}_{21}$ in terms of rmse, we can observe that $\% \mathrm{cv}$ is $74 \%$ versus a percentage equal to $98 \%$ generated sample in which the algorithm properly converge under $\mathrm{AGH}_{21}$ and to $\% 100$ under PseudoAGH $\mathrm{A}_{21}$. Moreover, as in all the previous cases, the latter results to be the fastest. We observe the same behavior of the different approximation methods also for $\rho=0.95$. In particular, in this case $\mathrm{GH}$ produces a $\% c v$ equal only to $36 \%$.

\subsection{Simulation study for the SV model}

The design of the study considered for the SV model is based on the same setting considered by Jacquier et al (1994) and widely used in the literature for evaluating the performance of different approximation methods. Here the results obtained with the AGH approximation are compared with those obtained by Fridman and Harris (1998), who used GLQ, by Bartolucci and De Luca (2001), who proposed RQ, and by Junji and Yoshihiko (2005), who used the Laplace approximation (LA). The Laplace approximation is considered here since it can be viewed as a particular case of the AGH when one quadrature point is chosen (Pinheiro and Bates, 1995).

The parameter values of the generating model were chosen so that different values of the $C V$ result. In more detail, the most relevant parameter $\rho$ is fixed to $0.90,0.95$ and 0.98 , values based on the empirical evidence. The other parameter values are consequently determined in such a way that $C V$ assumes the values $0.1,1.0$ and 10, producing nine different scenarios. In each simulated scenario, we let $T=500$ and used 500 Monte Carlo replications; for AGH we adopted $q=21$ quadrature points.

The mean of the parameter estimates and the root mean square error (rmse) in brackets for SV models with different combinations of the parameters are reported in Table 4.

[Table 4 about here.] 
The Table is grouped according to the values of the CV. We can observe that in the case of $\mathrm{CV}=10$ all the methods perform quite similarly in terms of rmse. Only in the case of $\rho=0.98$, GLQ produces better rmse than the other approximations. But, as the $\mathrm{CV}$ decreases, all the methods deteriorate sensibly apart from AGH that produces rmse always with the same small magnitude. The superiority of the proposed method is particularly noticeable in the case of $\mathrm{CV}=0.1$ for the parameters $\gamma$ and $\rho$. In terms of bias, AGH gives better or similar results than the other methods for most of the parameter values.

In the table, the average computational time (in seconds) of AGH are also showed for all the scenarios. We can observe that in all the cases the algorithm reaches convergence in few seconds. We cannot compare the computational performance of the proposed method with the other approximation methods since for the latter we do not have this information.

\section{Real data analysis}

In the following, we illustrate the proposed approach by two applications in the context of panel data analysis and in that of time-series data.

6.1 Application of limited dependent variable models to Self-reported health status

We consider panel data deriving from the Health and Retirement Study (HRS) 11 conducted by the University of Michigan with the aim of studying retirement and health among the elderly in the United States over time. These data are referred to a sample of $n=7,074$ individuals who were asked at $T=8$ time points (from 1992 to 2006 every two years) to report the self-rated health status (SHR) by answering to the question "Would you say your health is excellent, very good, good, fair, or poor?", that is an ordinal response variable with five categories. Also the covariates gender, race ("white", "non white"), education ("high school", "some college", "college and above"), and age measured at each time point are available. Some descriptive statistics for the distributions of the covariates are reported in Table 5 whereas Table 6 reports the conditional sample distribution of $\mathrm{SHR}_{t}$ on the previous response $\mathrm{SHR}_{t-1}$.

[Table 5 about here.]

[Table 6 about here.]

We observe that the sample is mainly composed by females $(58.1 \%)$, white individuals $(82.9 \%)$ with an average age at the first observed time point equal to 54.8 years. As for the level of education, $60.9 \%$ of the interviewed subjects declared to have a high-school diploma, $19.7 \%$ of them a college degree, and $19.4 \%$ a higher title.

\footnotetext{
1 The RAND HRS Data file is an easy to use longitudinal data set based on the HRS data. It was developed at RAND with funding from the National Institute on Aging and the Social Security Administration. See http://www.rand.org/labor/aging/dataprod.html for more details.
} 
The variable education has been recoded in the following way: 1 for "high school", 2 for "some college", and 3 for "college and above".

The high percentage of individuals (more than $50 \%$ percent for all the categories) that respond to the same category at time $t-1$ and at time $t$ indicates that SHR is highly correlated over time. Previous analysis on HRS data (Heiss, 2008) showed that the proportional odds model illustrated above with time dependent random intercepts following a stationary AR(1) well captures the SHR correlation pattern over time. More recently, Bartolucci et al (2014) analyzed the HRS data assuming a more flexible model based on a mixture of AR(1) for the latent process. They approximated the integrals with a rectangular quadrature method. Since here the aim is to evaluate the performance of the AGH discussed above, we fitted the proportional odds model with a standard stationary $\mathrm{AR}(1)$ using $\mathrm{AGH}_{21}$ and $\mathrm{PseudoAGH}_{21}$ approximation. For this particular sample, the algorithm under $\mathrm{GH}_{51}$ presented instability problems and hence it was not possible to compare the performance of the different approximations.

In Table 7 we report the results in terms of parameters estimates and correspondent standard errors under $\mathrm{AGH}_{21}$ and $\mathrm{Pseudo} \mathrm{AGH}_{21}$ approximation. The standard errors were obtained by the diagonal elements of the inverse of the observed information matrix.

[Table 7 about here.]

We observe that the parameter estimates are quite similar under PseudoAGH $\mathrm{AH}_{21}$ and $\mathrm{AGH}_{21}$ and in both cases all of them are significant apart from the coefficient of the covariate gender. It is worth noting that the autocorrelation parameter estimate is higher than 0.95 indicating a high persistent latent process over time. In this example, where the sample size is quite large, the computational time of PseudoAGH $\mathrm{A}_{21}$ is noticeably lower than that of $\mathrm{AGH}_{21}$.

\subsection{Application of stochastic volatility models to daily exchange rates}

To illustrate the application of the method to SV models, we use a data set analyzed by Harvey et al (1994) and later by several other authors. The data consist of a time series of daily pound/dollar exchange rates from the period October 1st, 1981 to June 28th, 1985. The series of interest is the logarithm of $n=945$ daily returns (Figure 4).

[Fig. 4 about here.]

For these data, we fitted the standard SV model illustrated above using GLQ, RQ, and AGH with $q=21$ quadrature points for approximating the integrals involved in the likelihood of the model. The results are reported in Table 8 . Under all the approximation methods $\rho$ has a high and significant value, indicating a highly persistent volatility process. However, it is worth noting that the variance estimate under $\mathrm{AGH}_{21}$ presents the lowest standard error and it is significantly different from 0 supporting the stochastic nature of the volatility process. On the contrary, GLQ and RQ variance estimates result not significantly different from 0 . 
[Table 8 about here.]

It is further interesting to compare the standard SV model with an SV model with error terms $\varepsilon_{i t}$ following a $t$-Student distribution with an unknown number of degrees of freedom $\nu$. This choice is motivated by the fact that many financial time series exhibit densities with fatter tails than the Normal distribution. For both specifications we used $\mathrm{AGH}_{21}$ given the better performance of this method in cases like this in which the estimated CV of the volatility is less than 1 . The results of parameter estimates are reported in Table 9

[Table 9 about here.]

Under both models, $\rho$ has a high and significant value. BIC, which is equal to 1859.05 for the SV Normal model versus 1862.36 for the SV $t$-Student model, suggests that there is no improvement assuming $t$-Student distributed errors.

The similarity between the two specifications of the error terms is confirmed by the estimated filtered volatilities obtained for each model. Figure 5 shows the plot of the estimated filtered volatilities for each time point of the $t$-Student distribution versus the Normal distribution. We observe that there is a substantial agreement in terms of estimated volatility between the two specifications.

[Fig. 5 about here.]

\section{Conclusions}

In this work we proposed the Adaptive Gauss Hermite (AGH) quadrature for approximating the integrals involved in the likelihood of a class of dynamic latent variable models based on a latent process following an autoregressive process of order 1, AR(1). In particular, we focused on Stochastic Volatility (SV) models for the analysis of financial time series and on Limited Dependent Variable (LDV) models for panel data. Both models can be formalized in a non-linear state space framework and maximum likelihood estimation can be obtained by mean of a non-linear filtering algorithm.

The main advantage of AGH compared with other numerical approximation methods is that it better captures the peak of the integrand in those cases in which it appears very sharp, using fewer quadrature points than other methods. This is due to the fact that the nodes of AGH are scaled and translated at each step of the algorithm with the posterior mean and the posterior standard deviation of the latent variables given the manifest variables.

The good behavior of AGH has been highlighted by means of simulation studies. For SV models we found that the advantages of AGH with respect to other methods are particularly evident in terms of parameter accuracy for those values of the model parameters that give low coefficients of variation of the volatility. For LDV, we found that the performance of AGH is related to the value of the autocorrelation parameter $\rho$. For high values of $\rho$, parameter estimates are very accurate under AGH even with just $q=15$ quadrature points whereas GH produces convergent solutions only in few cases when the sample size is large. On the other hand, for $\rho=0.5$, AGH 
gives results very similar to the classical GH but with a higher computational cost. To solve this problem, we considered a pseudo version of AGH that consists in updating the nodes of the quadrature only at the first step of the algorithm. We found a very good performance of this method in terms of both computational burden of the algorithm and parameter accuracy in almost all the scenarios we considered. However, differently from AGH, the performance of PseudoAGH depends on the choice of the starting values.

The potential of the proposed method has been showed also through two empirical examples, the first one referred to an American longitudinal survey on the health condition of the elderly population, the second one to a time series of daily pound/dollar exchange rates in a given period of time. In both examples, the latent variables showed high values of autocorrelation, values for which the adaptive quadrature presents the best performance compared with the other approximations. The high persistency of the latent variable is typical of SV models but it is also plausible in panel data for the underlying process of time dependent response variables, as for the health status considered here.

\section{Acknowledgements}

The authors acknowledge the financial support from the grant RBFR12SHVV funded by the Italian Government (FIRB project "Mixture and latent variable models for causal inference and analysis of socio-economic data").

\section{References}

Andersen T (1994) Stochastic autoregressive volatility: a framework for volatility modeling. Mathematical Finance 4:75-102

Bartolucci F, De Luca G (2001) Maximum likelihood estimation for a latent variable time series model. Applied Stochastic Models for Business and Industry 17:5-17

Bartolucci F, De Luca G (2003) Likelihood-based inference for asymmetric stochastic volatility models. Computational Statistical and Data Analysis 42:445-449

Bartolucci F, Farcomeni A, Pennoni F (2013) Latent Markov Models for Longitudinal Data. Chapman \& Hall/CRC Press, Boca Raton, FL

Bartolucci F, Bacci S, Pennoni F (2014) Longitudinal analysis of the self-reported health status by mixture latent autoregressive models. Journal of the Royal Statistical Society-series C 63:267-288

Bollerslev T (1986) Generalized autoregresive conditional heteroskedasticity. Journal of Econometrics 31:307-327

Cagnone S, Monari P (2013) Latent variable models for ordinal data by using the adaptive quadrature approximation. Computational Statistics 28:597-619

Durbin J, Koopman S (2002) A simple and efficient simulation smmother for space time series analyis. Biometrika 89:603-616

Engle R (1982) Autoregressive conditional heteroscedasticity with estimates of the variance of united kindom inflation. Econometrica 50:987-1007 
Fernández-Villaverde J, Rubio-Ramírez J (2006) Estimating macroeconomic models: A likelihood approach. Tech. rep., NBER Technical Working Paper 321

Frees E (2004) Longitudinal and Panel Data: Analysis and Application in Social Sciences

Fridman M, Harris L (1998) A maximum likelihood approach for non-gaussian stochastic volatility models. Journal of Business and Economic Statistics 16:284291

Hancock G, Samuelson K (2008) Advances in latent variable mixture models. Charlottee, NC: Information Age Publishing, United States of America

Harvey A, Ruiz E, Shephard N (1994) Multivariate stochastic variance models. Review of Economic Studies 61:247-264

Heiss F (2008) Sequential numerical integration in nonlinear state spece models for microeconometric panel. Journal of Applied Econometrics 23:373-389

Heiss F, Winschel V (2008) Likelihood approximation by numerical integration on sparse grids. Journal of Econometrics 144:62-80

Jacquier E, Polson N, Rossi P (1994) Bayesian analysis of stochastic volatility models. Journal of Business and Economic Statistics 12:371-417

Joe H (2008) Accuracy of laplace approximation for discrete response mixed models. Computational Statistics and Data Analysis 52:5066-5074

Junji S, Yoshihiko T (2005) Estimation of stochastic volatility models: an approximation to the nonlinear state space representation. Communications in StatisticsSimulation and Computation 34:429-450

Liu Q, Pierce D (1994) A note on gauss-hermite quadrature. Biometrika 81:624-629

McCullagh P (1980) Regression models for ordinal data (with discussion). Journal of the American Statistical Society, Series B 42:109-142

Naylor J, Smith A (1982) Applications of a method for the efficient computation of posterior distributions. Applied Statistics 51:214-225

Pinheiro J, Bates D (1995) Approximation to the loglikelihood function in the nonlinear mixed effects model. Journal of Computational and Graphical Statistics 4:12 35

Rabe-Hesketh S, Skrondal A, Pickles A (2002) Reliable estimation of generalized linear mixed models using adaptive quadrature. The Stata Journal 2:1:21

Rabe-Hesketh S, Skrondal A, Pickles A (2005) Maximum likelihood estimation of limited and discrete dependent variable models with nested random effects. Journal of Econometrics 128:301-323

Rizopoulus D (2012) Fast fitting of joint models for longitudinal and event time data using a pseudo-adaptive gaussian quadrature rule. Computational Statistics and Data Analysis 56:491-501

Schilling S, Bock R (2005) High-dimensional maximum marginal likelihood item factor analysis by adaptive quadrature. Psychometrika 70:533-555

Skrondal A, Rabe-Hesketh S (2004) Generalized Latent Variable Modeling. Multilevel, Longitudinal and Structural Equation Models. Sage Publication, London

Tanizaki H, Mariano R (1998) Nonlinear and nonnormal state-space modeling with monte-carlo stochastic simulations. Journal of Econometrics 83:263-290

Taylor S (1986) Modelling Financial Time Series. New York: Wiley

Taylor S (1994) Modeling stochastic volatility. Mathematical Finance pp 183-204 

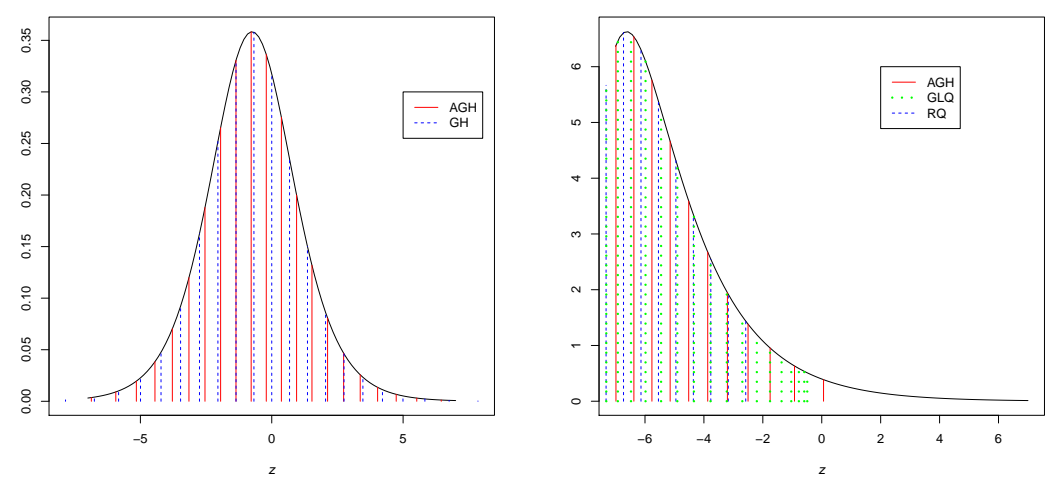

Fig. 1 Approximating $f_{y}\left(y_{1} \mid \alpha_{1}\right)$ for the LDV model (left picture) and SV model (right picture)
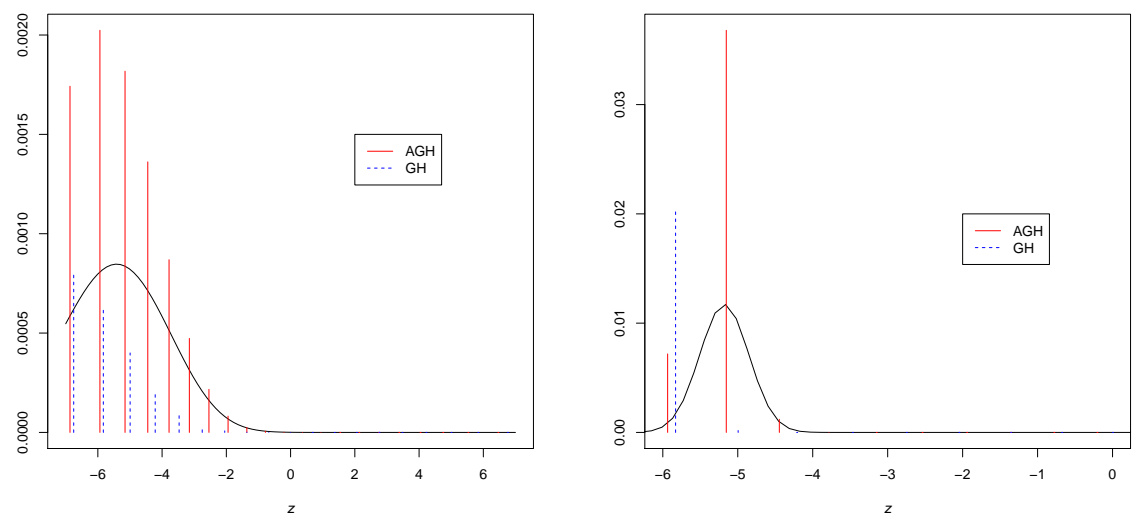

Fig. 2 Approximating $\phi\left(\alpha_{2} ; \rho \alpha_{1},\left(1-\rho^{2}\right)\right) f_{y}\left(y_{1} \mid \alpha_{1}\right)$ for the LDV model, $\rho=0.5$ (left picture) and $\rho=0.95$ (right picture) 

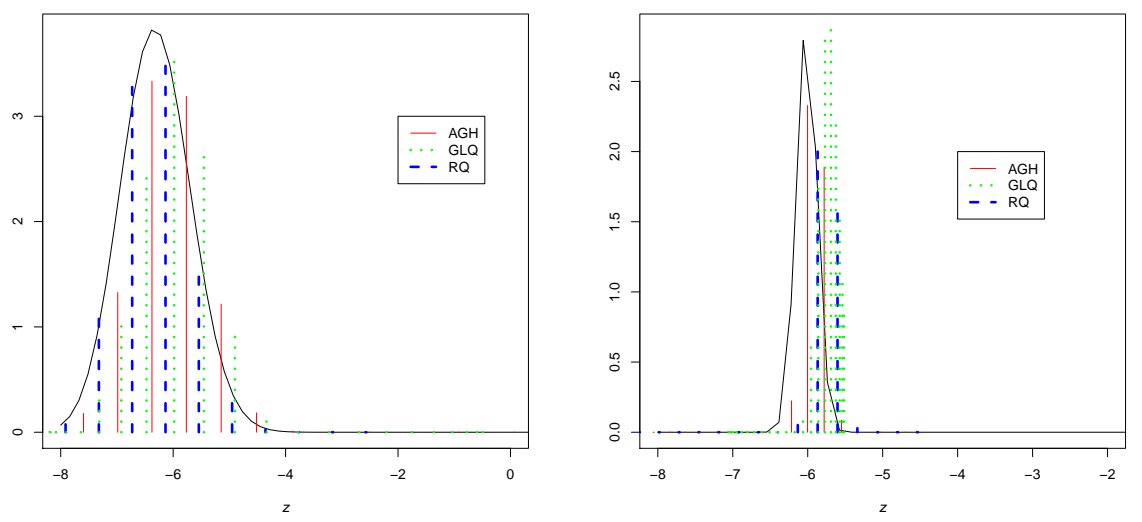

Fig. 3 Approximating $\phi\left(\alpha_{2} ; \gamma+\rho \alpha_{1}, \sigma_{\eta}^{2}\right) f_{y}\left(y_{1} \mid \alpha_{1}\right)$ for the $S V$ model, $C V=10$ (left picture) and $C V=0.1$ (right picture)

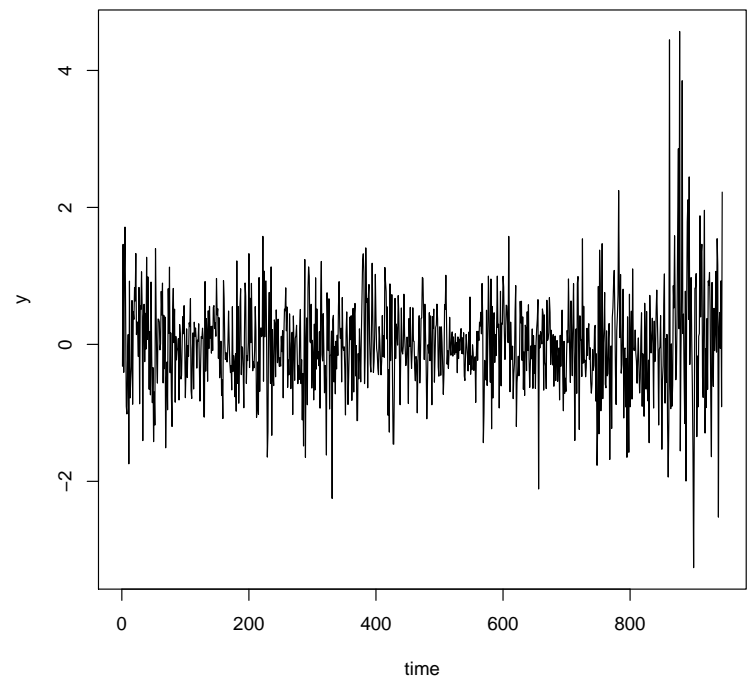

Fig. 4 Log-daily difference of the pound-dollar exchange rate from October 1st, 1981 to June 28th, 1985. 


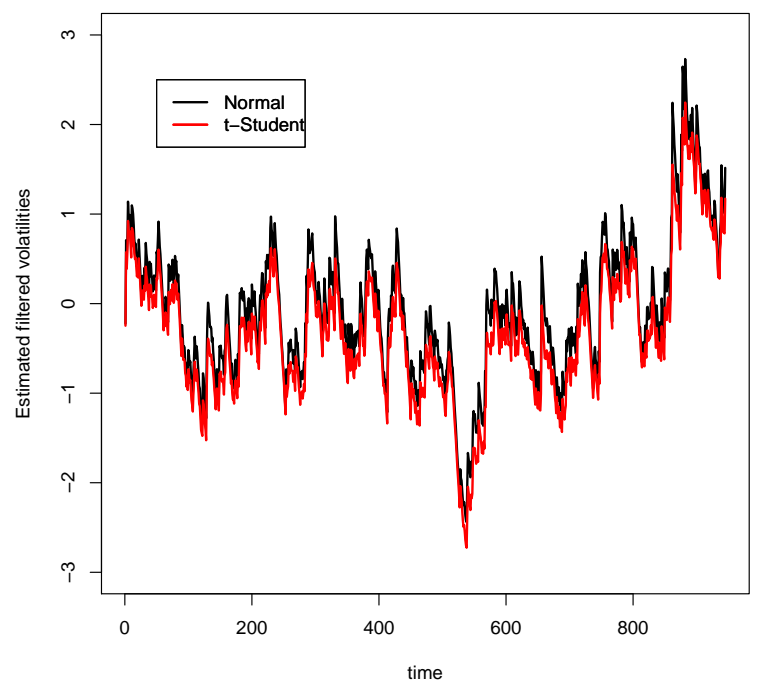

Fig. 5 Filtered estimated volatilities obtained assuming the Normal distribution and the $t$-Student distribution for the error term for the daily exchange rates dataset. 
Table 1 Estimated mean and rmse (in brackets) for the LDV model parameters under GH and AGH for $T=5, n=500$; results based on 500 replications.

\begin{tabular}{cccccc}
\hline \hline True value & $G_{21}$ & $G H_{51}$ & $A G H_{15}$ & $A G H_{21}$ & PseudoAGH $H_{21}$ \\
\hline$\rho=0.5$ & 0.478 & 0.487 & 0.487 & 0.492 & 0.493 \\
& $(0.17)$ & $(0.18)$ & $(0.18)$ & $(0.19)$ & $(0.19)$ \\
$\beta=1$ & 1.109 & 1.094 & 1.118 & 1.087 & 1.100 \\
& $(0.35)$ & $(0.28)$ & $(0.40)$ & $(0.25)$ & $(0.34)$ \\
$\sigma=1$ & 1.271 & 1.238 & 1.287 & 1.216 & 1.244 \\
& $(0.86)$ & $(0.72)$ & $(0.97)$ & $(0.67)$ & $(0.84)$ \\
$\% c v$ & 92 & 95 & 99 & 100 & 100 \\
nrfeval & 48 & 48 & 41 & 41 & 40 \\
av time $(\mathrm{sec})$ & 11.14 & 40.36 & 78.36 & 125.54 & 32.26 \\
\hline$\rho=0.90$ & - & - & 0.871 & 0.884 & 0.882 \\
& & & $(0.05)$ & $(0.05)$ & $(0.05)$ \\
$\beta=1$ & - & - & 1.016 & 1.011 & 1.012 \\
& & & $(0.05)$ & $(0.05)$ & $(0.05)$ \\
$\sigma=1$ & - & - & 1.045 & 1.028 & 1.030 \\
& & & $(0.10)$ & $(0.11)$ & $(0.10)$ \\
$\% c v$ & - & - & 100 & 100 & 100 \\
nrfeval & - & - & 28 & 35 & 31 \\
av time $(\mathrm{sec})$ & - & - & 64.91 & 129.56 & 22.68 \\
\hline$\rho=0.95$ & - & - & 0.898 & 0.917 & 0.914 \\
& - & - & $(0.06)$ & $(0.05)$ & $(0.03)$ \\
$\beta=1$ & - & - & 1.025 & 1.015 & 1.019 \\
& - & - & $(0.05)$ & $(0.05)$ & $(0.05)$ \\
$\sigma=1$ & - & - & 1.074 & 1.050 & 1.053 \\
& - & - & $(0.11)$ & $(0.10)$ & $(0.10)$ \\
\%cv & - & - & 100 & 100 & 100 \\
nrfeval & - & - & 27 & 32 & 28 \\
av time $(\mathrm{sec})$ & - & - & 65.4 & 100.18 & 19.14 \\
\hline & & & & &
\end{tabular}


Table 2 Estimated mean and rmse (in brackets) for the LDV model parameters under GH and AGH, $T=10, n=500$; results based on 500 replications.

\begin{tabular}{|c|c|c|c|c|c|}
\hline True value & $G H_{21}$ & $G H_{51}$ & $A G H_{15}$ & $A G H_{21}$ & Pseudo $\mathrm{AGH}_{21}$ \\
\hline \multirow[t]{2}{*}{$\rho=0.5$} & 0.455 & 0.481 & 0.490 & 0.490 & 0.490 \\
\hline & $(0.10)$ & $(0.10)$ & $(0.10)$ & $(0.10)$ & $(0.10)$ \\
\hline \multirow[t]{2}{*}{$\beta=1$} & 1.044 & 1.028 & 1.025 & 1.023 & 1.025 \\
\hline & $(0.11)$ & $(0.11)$ & $(0.14)$ & $(0.10)$ & $(0.13)$ \\
\hline \multirow[t]{2}{*}{$\sigma=1$} & 1.137 & 1.081 & 1.070 & 1.065 & 1.070 \\
\hline & $(0.31)$ & $(0.30)$ & $(0.35)$ & $(0.29)$ & $(0.34)$ \\
\hline \multirow{3}{*}{$\begin{array}{c}\% c v \\
n r \text { feval } \\
\text { av time (sec) }\end{array}$} & 61 & 81 & 100 & 100 & 100 \\
\hline & 63 & 53 & 56 & 56 & 56 \\
\hline & 24.43 & 80.12 & 227.48 & 288.38 & 73.23 \\
\hline \multirow[t]{2}{*}{$\rho=0.90$} & - & - & 0.892 & 0.898 & 0.898 \\
\hline & - & - & $(0.02)$ & $(0.02)$ & $(0.02)$ \\
\hline \multirow[t]{2}{*}{$\beta=1$} & - & - & 1.003 & 1.001 & 1.001 \\
\hline & - & - & $(0.03)$ & $(0.03)$ & $(0.03)$ \\
\hline \multirow[t]{2}{*}{$\sigma=1$} & - & - & 1.011 & 1.001 & 1.002 \\
\hline & - & - & $(0.06)$ & $(0.06)$ & $(0.06)$ \\
\hline$\% c v$ & - & - & 97 & 98 & 100 \\
\hline$n r$ feval & - & - & 37 & 36 & 36 \\
\hline av time ( $\mathrm{sec})$ & - & - & 137.64 & 188.03 & 37.91 \\
\hline True value & $\mathrm{GH}_{21}$ & $\mathrm{GH}_{51}$ & $A G H_{15}$ & $A G H_{21}$ & Pseudo $\mathrm{AGH}_{21}$ \\
\hline \multirow[t]{2}{*}{$\rho=0.95$} & - & - & 0.923 & 0.938 & 0.935 \\
\hline & - & - & $(0.03)$ & $(0.02)$ & $(0.04)$ \\
\hline \multirow[t]{2}{*}{$\beta=1$} & - & - & 1.013 & 1.007 & 1.010 \\
\hline & - & - & $(0.04)$ & $(0.04)$ & $(0.03)$ \\
\hline \multirow[t]{2}{*}{$\sigma=1$} & - & - & 1.034 & 1.017 & 1.030 \\
\hline & - & - & $(0.15)$ & $(0.08)$ & $(0.06)$ \\
\hline$\% c v$ & - & - & 99 & 97 & 100 \\
\hline$n r$ feval & - & - & 36 & 36 & 39 \\
\hline av time $(\mathrm{sec})$ & - & - & 164.71 & 278.73 & 52.23 \\
\hline
\end{tabular}


Table 3 Estimated mean and rmse (in brackets) for the LDV model parameters under GH and AGH for $T=5, n=1000$; results based on 500 replications.

\begin{tabular}{|c|c|c|c|c|c|}
\hline True value & $\mathrm{GH}_{21}$ & $G H_{51}$ & $A G H_{15}$ & $A G H_{21}$ & Pseudo $A G H_{21}$ \\
\hline \multirow[t]{2}{*}{$\rho=0.5$} & 0.478 & 0.482 & 0.487 & 0.486 & 0.488 \\
\hline & $(0.14)$ & $(0.14)$ & $(0.14)$ & $(0.15)$ & $(0.14)$ \\
\hline \multirow[t]{2}{*}{$\beta=1$} & 1.064 & 1.058 & 1.058 & 1.053 & 1.057 \\
\hline & $(0.19)$ & $(0.17)$ & $(0.23)$ & $(0.16)$ & $(0.23)$ \\
\hline \multirow[t]{2}{*}{$\sigma=1$} & 1.170 & 1.150 & 1.147 & 1.138 & 1.145 \\
\hline & $(0.52)$ & $(0.48)$ & $(0.58)$ & $(0.46)$ & $(0.58)$ \\
\hline$\% c v$ & 77 & 92 & 100 & 100 & 100 \\
\hline \multirow{2}{*}{$\begin{array}{c}\text { nrfeval } \\
\text { av time }(\mathrm{sec})\end{array}$} & 50 & 55 & 58 & 57 & 57 \\
\hline & 24.46 & 83.27 & 191.66 & 309.6 & 72.99 \\
\hline \multirow[t]{2}{*}{$\rho=0.90$} & 0.828 & 0.876 & 0.875 & 0.888 & 0.887 \\
\hline & $(0.08)$ & $(0.04)$ & $(0.04)$ & $(0.04)$ & $(0.04)$ \\
\hline \multirow[t]{2}{*}{$\beta=1$} & 1.030 & 1.013 & 1.013 & 1.008 & 1.009 \\
\hline & $(0.05)$ & $(0.04)$ & $(0.04)$ & $(0.03)$ & $(0.03)$ \\
\hline \multirow[t]{2}{*}{$\sigma=1$} & 1.099 & 1.038 & 1.036 & 1.020 & 1.021 \\
\hline & $(0.12)$ & $(0.08)$ & $(0.07)$ & $(0.08)$ & $(0.07)$ \\
\hline$\% c v$ & 18 & 74 & 96 & 98 & 100 \\
\hline \multirow{2}{*}{$\begin{array}{c}n r f e v a l \\
\text { av time }(\mathrm{sec})\end{array}$} & 55 & 50 & 49 & 50 & 50 \\
\hline & 19.36 & 70.84 & 167.64 & 312.05 & 59.84 \\
\hline \multirow[t]{2}{*}{$\rho=0.95$} & - & 0.902 & 0.901 & 0.923 & 0.918 \\
\hline & - & $(0.05)$ & $(0.05)$ & $(0.04)$ & $(0.04)$ \\
\hline \multirow[t]{2}{*}{$\beta=1$} & - & 1.021 & 1.022 & 1.015 & 1.016 \\
\hline & - & $(0.04)$ & $(0.04)$ & $(0.05)$ & $(0.03)$ \\
\hline \multirow[t]{2}{*}{$\sigma=1$} & - & 1.067 & 1.068 & 1.036 & 1.046 \\
\hline & - & (0.09) & $(0.12)$ & $(0.10)$ & (0.07) \\
\hline$\% c v$ & - & 36 & 98 & 100 & 100 \\
\hline nrfeval & - & 50 & 44 & 48 & 46 \\
\hline av time $(\mathrm{sec})$ & - & 72.49 & 192.63 & 269.41 & 62.11 \\
\hline
\end{tabular}


Table 4 Comparison between different approximation methods in terms of the estimated means and rmse of the SV model parameters.

\begin{tabular}{|c|c|c|c|c|c|c|c|c|c|}
\hline $\begin{array}{l}\text { CV }=10 \\
\text { TRUE }\end{array}$ & $\begin{array}{c}\gamma \\
-0.821\end{array}$ & $\begin{array}{c}\rho \\
0.90\end{array}$ & $\begin{array}{c}\sigma_{\eta} \\
0.675\end{array}$ & $\begin{array}{c}\gamma \\
-0.411\end{array}$ & $\begin{array}{c}\rho \\
0.95\end{array}$ & $\begin{array}{c}\sigma_{\eta} \\
0.484\end{array}$ & $\begin{array}{c}\gamma \\
-0.164\end{array}$ & $\begin{array}{c}\rho \\
0.98\end{array}$ & $\begin{array}{c}\sigma_{\eta} \\
0.308\end{array}$ \\
\hline \multirow[t]{2}{*}{ GLQ } & -0.896 & 0.890 & 0.685 & -0.505 & 0.940 & 0.495 & -0.100 & 0.986 & 0.320 \\
\hline & $(0.28)$ & $(0.03)$ & $(0.08)$ & $(0.18)$ & $(0.02)$ & $(0.07)$ & $(0.08)$ & $(0.01)$ & $(0.05)$ \\
\hline \multirow[t]{2}{*}{ RQ } & -0.859 & 0.895 & 0.694 & -0.472 & 0.943 & 0.503 & -0.275 & 0.967 & 0.343 \\
\hline & $(0.25)$ & $(0.03)$ & $(0.08)$ & $(0.18)$ & $(0.02)$ & $(0.07)$ & $(0.18)$ & $(0.02)$ & $(0.07)$ \\
\hline \multirow[t]{2}{*}{ LA } & -0.905 & 0.880 & 0.727 & -0.510 & 0.931 & 0.534 & -0.259 & 0.965 & 0.343 \\
\hline & $(0.28)$ & $(0.04)$ & $(0.10)$ & $(0.23)$ & $(0.03)$ & (0.09) & $(0.18)$ & $(0.02)$ & $(0.07)$ \\
\hline \multirow[t]{2}{*}{$\mathrm{AGH}$} & -0.613 & 0.925 & 0.725 & -0.509 & 0.938 & 0.527 & -0.276 & 0.966 & 0.358 \\
\hline & $(0.23)$ & $(0.03)$ & $(0.15)$ & $(0.16)$ & $(0.02)$ & $(0.08)$ & $(0.19)$ & $(0.02)$ & $(0.08)$ \\
\hline av time (sec) & & 17.97 & & & 12.34 & & & 14.86 & \\
\hline $\mathrm{CV}=1.0$ & $\gamma$ & $\rho$ & $\sigma_{\eta}$ & $\gamma$ & $\rho$ & $\sigma_{\eta}$ & $\gamma$ & $\rho$ & $\sigma_{\eta}$ \\
\hline TRUE & -0.736 & 0.90 & 0.363 & -0.368 & 0.95 & 0.26 & -0.147 & 0.98 & 0.166 \\
\hline \multirow[t]{2}{*}{ GLQ } & -0.870 & 0.880 & 0.370 & -0.510 & 0.930 & 0.280 & -0.090 & 0.987 & 0.180 \\
\hline & $(0.43)$ & $(0.05)$ & $(0.08)$ & $(0.31)$ & $(0.04)$ & $(0.07)$ & $(0.06)$ & $(0.02)$ & $(0.04)$ \\
\hline \multirow[t]{2}{*}{ RQ } & -0.812 & 0.890 & 0.375 & -0.492 & 0.933 & 0.278 & -0.308 & 0.958 & 0.214 \\
\hline & $(0.45)$ & $(0.06)$ & (0.09) & $(0.29)$ & $(0.04)$ & $(0.07)$ & $(0.25)$ & $(0.03)$ & $(0.08)$ \\
\hline \multirow[t]{2}{*}{ LA } & -0.926 & 0.872 & 0.422 & -0.526 & 0.927 & 0.303 & -0.278 & 0.961 & 0.200 \\
\hline & $(0.42)$ & $(0.06)$ & $(0.11)$ & $(0.39)$ & $(0.05)$ & $(0.10)$ & $(0.25)$ & $(0.03)$ & $(0.07)$ \\
\hline \multirow[t]{2}{*}{$\mathrm{AGH}$} & -0.572 & 0.922 & 0.359 & -0.475 & 0.935 & 0.293 & -0.341 & 0.953 & 0.213 \\
\hline & $(0.17)$ & $(0.02)$ & $(0.04)$ & $(0.21)$ & $(0.03)$ & $(0.08)$ & $(0.26)$ & $(0.03)$ & $(0.07)$ \\
\hline av time (sec) & & 13.85 & & & 11.59 & & & 17.83 & \\
\hline $\mathrm{CV}=0.1$ & $\gamma$ & $\rho$ & $\sigma_{\eta}$ & $\gamma$ & $\rho$ & $\sigma_{\eta}$ & $\gamma$ & $\rho$ & $\sigma_{\eta}$ \\
\hline TRUE & -0.706 & 0.90 & 0.135 & -0.353 & 0.95 & 0.096 & -0.141 & 0.98 & 0.061 \\
\hline \multirow[t]{2}{*}{ GLQ } & -1.360 & 0.810 & 0.160 & -0.810 & 0.886 & 0.120 & -0.537 & 0.924 & 0.088 \\
\hline & (1.72) & $(0.24)$ & $(0.12)$ & (1.15) & $(0.16)$ & $(0.09)$ & (1.13) & $(0.16)$ & $(0.09)$ \\
\hline \multirow[t]{2}{*}{ RQ } & 0.944 & 0.873 & 0.159 & -0.796 & 0.888 & 0.148 & -0.515 & 0.927 & 0.122 \\
\hline & $(1.24)$ & $(0.17)$ & $(0.10)$ & $(0.77)$ & $(0.11)$ & $(0.10)$ & $(0.94)$ & $(0.13)$ & $(0.11)$ \\
\hline \multirow[t]{2}{*}{ LA } & -1.227 & 0.827 & 0.178 & -0.763 & 0.892 & 0.133 & -0.489 & 0.93 & 0.099 \\
\hline & $(1.55)$ & $(0.22)$ & $(0.14)$ & (1.16) & $(0.16)$ & $(0.12)$ & $(0.98)$ & $(0.14)$ & $(0.11)$ \\
\hline \multirow[t]{2}{*}{$\mathrm{AGH}$} & -0.521 & 0.926 & 0.137 & -0.455 & 0.935 & 0.098 & -0.568 & 0.920 & 0.131 \\
\hline & $(0.21)$ & $(0.03)$ & $(0.08)$ & $(0.18)$ & $(0.03)$ & $(0.08)$ & $(0.52)$ & $(0.07)$ & $(0.10)$ \\
\hline av time $(\mathrm{sec})$ & & 17.02 & & & 20.03 & & & 18.77 & \\
\hline
\end{tabular}


Table 5 Summary statistics for the covariates in the HRS dataset; $n=7074$.

\begin{tabular}{lcc}
\hline Variable & Mean & Stdev \\
\hline Gender (female) & 0.581 & 0.490 \\
Race (nonwhite) & 0.171 & 0.377 \\
Education & & \\
$\quad$ (high school) & 0.609 & 0.488 \\
$\quad$ (college degree) & 0.197 & 0.398 \\
$\quad$ (college +) & 0.194 & 0.395 \\
Age $_{92}$ & 54.80 & 5.460 \\
\hline
\end{tabular}

Table 6 Conditional distribution of SHR given SHR $t-1$ for the HRS dataset; $n=7074$.

\begin{tabular}{lrrrrr}
\hline & poor & fair & good & very good & excellent \\
\hline poor & 54.5 & 34.1 & 8.4 & 2.5 & 0.7 \\
fair & 12.8 & 51.0 & 27.4 & 7.2 & 1.6 \\
good & 2.5 & 16.5 & 53.3 & 23.6 & 4.1 \\
very good & 0.8 & 4.7 & 25.9 & 55.6 & 13.0 \\
excellent & 0.4 & 1.9 & 10.6 & 33.7 & 53.4 \\
\hline
\end{tabular}

Table 7 Estimates of the parameters of the LDV model adopted for the analysis of the HRS dataset (standard errors in brackets).

\begin{tabular}{lcc}
\hline & $A G H$ & PseudoAGH \\
\hline$\hat{\beta}_{1}$ female & -0.147 & -0.099 \\
& $(0.074)$ & $(0.073)$ \\
$\hat{\beta}_{2}$ non white & -1.509 & -1.394 \\
& $(0.096)$ & $(0.091)$ \\
$\hat{\beta}_{3}$ education & 1.182 & 1.141 \\
$\hat{\beta}_{4}$ age & $(0.046)$ & $(0.047)$ \\
& -0.109 & -0.089 \\
& $(0.003)$ & $(0.003)$ \\
$\hat{\rho}$ & 0.953 & 0.955 \\
& $(0.018)$ & $(0.015)$ \\
$\hat{\sigma}$ & 3.121 & 2.860 \\
& $(0.036)$ & $(0.034)$ \\
Log-lik & -63591.17 & -63595.50 \\
Time $(\mathrm{sec})$ & 9612.34 & 2588.60 \\
\hline & & \\
\hline
\end{tabular}


Table 8 Estimate of the parameters of SV model adopted of the analysis of the daily exchange rates using $G L Q, R Q$ and $A G H_{21}$ methods(with standard errors in brackets).

\begin{tabular}{cccc}
\hline & GLQ & RQ & $\mathrm{AGH}_{21}$ \\
\hline$\gamma$ & -0.016 & -0.025 & -0.033 \\
& $(0.009)$ & $(0.014)$ & $(0.012)$ \\
$\rho$ & 0.982 & 0.974 & 0.964 \\
& $(0.013)$ & $(0.020)$ & $(0.010)$ \\
$\sigma_{\eta}^{2}$ & 0.027 & 0.023 & 0.039 \\
& $(0.047)$ & $(0.046)$ & $(0.001)$ \\
\hline
\end{tabular}

Table 9 Estimate of the parameters of SV model adopted of the analysis of the daily exchange rates using $A G H_{21}$ (with standard errors in brackets).

\begin{tabular}{lll}
\hline & \multicolumn{2}{c}{ Model } \\
\hline & SV Normal & SV $t$-Student \\
\hline$\gamma$ & -0.033 & -0.015 \\
& $(0.012)$ & $(0.010)$ \\
$\rho$ & 0.964 & 0.966 \\
& $(0.010)$ & $(0.001)$ \\
$\sigma_{\eta}^{2}$ & 0.039 & 0.034 \\
& $(0.001)$ & $(0.001)$ \\
$\nu$ & - & 25.97 \\
& & $(11.92)$ \\
& & \\
Log-lik & -919.25 & -917.49 \\
BIC & 1859.05 & 1862.37 \\
Time (sec) & 29.73 & 34.79 \\
\hline
\end{tabular}

